\title{
A percepção do controle dos sintomas em pacientes asmáticos*
}

\author{
Perception of asthma control in asthma patients \\ Eduardo Vieira Ponte 1 , Jaqueline Petroni ${ }^{2}$, Daniela Campos Borges Ramos ${ }^{3}$,
Luciana Pimentel ${ }^{3}$, Daise Naiane Freitas ${ }^{4}$, Álvaro A Cruz
}

Resumo

Objetivo: Estimar a proporção de pacientes asmáticos com percepção inadequada do controle dos sintomas e avaliar as características deste subgrupo. Métodos: Estudo transversal no qual foram selecionados pacientes ambulatoriais consecutivos com asma leve, moderada ou grave, maiores de 12 anos. Os pacientes foram submetidos a exame clínico, avaliação de depressão e da percepção do controle da asma e exame de função pulmonar. Foram realizadas avaliação da concordância quanto ao controle dos sintomas de asma entre médicos e pacientes e comparação entre as características dos pacientes com percepção adequada e inadequada do controle da asma. Resultados: A concordância entre médicos e pacientes quanto ao controle da asma foi baixa (índice kappa =0,5). Dos 289 pacientes incluídos, 66 (23\%) apresentaram percepção inadequada do controle da asma. A análise preliminar univariada revelou que os pacientes com percepção inadequada eram mais idosos, tinham menor renda familiar e apresentavam asma de menor gravidade. A análise multivariada não modificou os resultados. Não houve diferença entre os grupos quanto a sexo, freqüência de pacientes alfabetizados, tempo de sintomas de asma, diagnóstico de rinite, freqüência de depressão, função pulmonar ou adesão ao tratamento. Conclusões: A freqüência de percepção inadequada do controle da doença em pacientes com asma é elevada, principalmente em pacientes idosos, com baixa renda familiar e asma de menor gravidade.

Descritores: Asma; Sinais e sintomas; Asma/terapia.

\begin{abstract}
Objective: To determine the proportion of asthma patients with poor perception of asthma control and to evaluate the characteristics of this subgroup. Methods: A cross-sectional study in which consecutive outpatients (over the age of 12) with mild, moderate, or severe asthma were selected. The patients underwent clinical examination, and pulmonary function tests, as well as being assessed for depression and perception of asthma control. The degree of concordance between patients and physicians regarding the perception of asthma control was determined. Patients with good perception of asthma control were compared, in terms of characteristics, with those presenting poor perception. Results: The degree of concordance between patients and physicians regarding the perception of asthma control was low (kappa index $=0.5)$. Of the 289 patients included, $66(23 \%)$ presented poor perception of asthma control. The preliminary univariate analysis revealed that the patients with poor perception of asthma control were older, had a lower income, and presented a lower degree of asthma severity. Those factors did not change in the multivariate analysis. There were no differences between the groups in terms of gender, frequency of having literate patients, duration of asthma symptoms, having been diagnosed with rhinitis, frequency of depression, pulmonary function, or treatment compliance. Conclusions: The incidence of poor perception of asthma control in asthma patients is high, especially in elderly patients with lower income and a lower degree of asthma severity.
\end{abstract}

Keywords: Asthma; Signs and symptoms; Asthma/therapy.

\footnotetext{
* Trabalho realizado pelo Programa de Controle da Asma e da Rinite Alérgica na Bahia - ProAR - Universidade Federal da Bahia - UFBA - Salvador (BA) Brasil. 1. Doutorando do Programa de Pós-Graduação em Medicina e Saúde. Hospital Universitário Professor Edgard Santos - HUPES - Universidade Federal da Bahia UFBA - Salvador (BA) Brasil.

2. Professora de Fisioterapia. Universidade Católica de Salvador - UCSAL - Salvador (BA) Brasil.

3. Acadêmica de Medicina. Universidade Federal da Bahia - UFBA - Salvador (BA) Brasil.

4. Acadêmica de Enfermagem. Universidade Católica de Salvador - UCSAL - Salvador (BA) Brasil.

5. Coordenador Geral do Programa de Controle da Asma e da Rinite Alérgica na Bahia ProAR - Universidade Federal da Bahia - UFBA - Salvador (BA) Brasil.

Endereço para correspondência: Eduardo Vieira Ponte. Programa de Controle da Asma e da Rinite Alérgica na Bahia - ProAR, Rua Carlos Gomes, 270, $7^{\circ}$ andar, Centro Médico Carlos Gomes, CEP 40060-330, Salvador, BA, Brasil.

Tel 5571 203-2749. E-mail: evponte@yahoo.com.br

Recebido para publicação em 10/9/2006. Aprovado, após revisão, em 27/2/2007.
} 


\section{Introdução}

0 objetivo do tratamento da asma é o controle total dos sintomas. lsso pode ser obtido com o uso combinado de corticóide inalatório e beta- 2 agonista de longa ação, até mesmo em pacientes com sintomas de asma grave. ${ }^{(1)}$ Apesar disso, um estudo epidemiológico realizado em sete centros europeus demonstrou que um percentual elevado de pacientes asmáticos não atinge o controle adequado dos sintomas. ${ }^{(2)} \mathrm{A}$ falta de adesão ao tratamento é uma das causas do controle inadequado da asma, mesmo quando o paciente está sob os cuidados do especialista. ${ }^{(3)}$

Um outro fator que pode contribuir para a falta de controle da asma é a prescrição de medicações preventivas em doses insuficientes. 0 programa conhecido como Detection, Intervention, and Monitoring of COPD and Asthma demonstrou que $66 \%$ dos pacientes com asma não informaram espontaneamente os sintomas ao médico. ${ }^{(4)}$ Conseqüentemente, o médico pode subestimar a gravidade da doença, prescrevendo um tratamento inadequado. Por outro lado, alguns pacientes com sintomas não relacionados à asma podem referir que a doença não está completamente controlada, o que pode levar o médico a superestimar a gravidade da doença, prescrevendo tratamento excessivo. Portanto, a percepção do controle da asma pelo paciente tem implicações muito diretas no manejo da doença pelo médico, o qual orienta o tratamento principalmente com base nos sintomas referidos.

Em Salvador, o Programa de Controle da Asma e da Rinite Alérgica da Bahia (ProAR) oferece atendimento especializado e tratamento gratuito para pacientes com asma grave desde o ano de 2002. Atualmente, 1.605 pacientes estão incluídos no programa, sendo que 1.070 pacientes estão em acompanhamento no ambulatório do ProAR da Universidade Federal da Bahia. Nestes pacientes, houve uma redução de 10 vezes no número de atendimentos em emergência e de internações hospitalares por crise de broncoespasmo. ${ }^{(5)}$

0 objetivo do presente estudo, no qual foram avaliados pacientes do ProAr e do ambulatório de asma do Hospital Universitário Professor Edgard Santos, foi determinar a proporção de pacientes com asma que percebem de forma inadequada o controle da doença, estudar as características que diferenciam os indivíduos com percepção adequada daqueles com percepção inadequada do controle da asma e avaliar se a percepção do controle da asma influencia a adesão ao tratamento.

\section{Métodos}

Em uma avaliação transversal, foram selecionados pacientes consecutivos com diagnóstico de asma leve e moderada do ambulatório do Hospital Universitário Professor Edgard Santos (Universidade Federal da Bahia) e pacientes com asma grave do ProAR. Este foi o único critério de inclusão do presente estudo. Foram excluídos apenas os pacientes que se recusaram a assinar o termo de consentimento livre e esclarecido. Os pacientes foram submetidos a avaliação clínica, avaliação de depressão, avaliação da percepção do controle da asma e exame de função pulmonar. 0 estudo foi aprovado pelo Comitê de Ética em Pesquisa da instituição.

A avaliação clínica foi realizada por um médico pneumologista. Foram colhidas informações sobre características demográficas e socioeconômicas, tempo de sintomas de asma, medicações usadas no último mês e presença de sintomas de rinite. Além disso, foi realizada a classificação da gravidade da asma de acordo com os critérios do III Consenso Brasileiro no Manejo da Asma. ${ }^{(6)}$ Os pacientes que estavam em acompanhamento no ProAR e apresentavam sintomas não controlados foram questionados pelo médico se haviam feito uso diário de corticóide inalatório no último mês. Neste programa, os pacientes recebem medicação gratuita. 0 relato do paciente de haver feito uso diário do corticóide inalatório no último mês foi considerado um indicador de adesão ao tratamento. A avaliação da adesão ao tratamento não foi feita nos pacientes não participantes do ProAR pois a renda familiar muito baixa da população estudada determina uma freqüência muito baixa de adesão ao tratamento pela falta de acesso às medicações.

0 paciente e o médico classificaram, de forma independente, o controle da asma nos últimos sete dias em 'totalmente controlada', 'bem controlada', 'parcialmente controlada', 'mal controlada' e 'totalmente descontrolada'. A asma foi considerada controlada quando um dos dois primeiros itens foi selecionado. Quando um dos três últimos itens foi selecionado, a asma foi considerada não controlada. 0 médico definiu o controle dos sintomas 
de asma com base em informações obtidas pelos exames clínico e de função pulmonar. A presença de sintomas persistentes nos últimos sete dias foi utilizada como critério para o médico classificar a asma em não controlada (parcialmente controlada, mal controlada ou totalmente descontrolada). A presença de sintomas intermitentes ou ausentes nos últimos sete dias foi o parâmetro utilizado pelo médico para classificar a asma em controlada (totalmente controlada ou bem controlada). 0 paciente foi orientado a definir o controle da asma de acordo com a impressão pessoal, baseado no histórico da sua doença nos últimos sete dias.

O Inventário de Depressão de Beck, versão II, foi utilizado para classificar os pacientes quanto à presença de depressão. Devido ao percentual elevado de pacientes com baixa escolaridade, o questionário foi aplicado por um entrevistador. 0 entrevistador foi orientado a não interpretar as perguntas do questionário e a não influenciar as respostas do paciente. 0 escore do questionário pode variar de 0 a 63. Pacientes com escore acima de 19 foram considerados deprimidos. ${ }^{(7,8)}$

As espirometrias foram realizadas com espirômetro KoKo (PDS Instrumentation, Inc., Louisville, Colorado, EUA), de acordo com as normas da Sociedade Brasileira de Pneumologia e Tisiologia, ${ }^{\left({ }^{9}\right.}$ usando os parâmetros de normalidade para a população brasileira. 0 volume expiratório forçado no primeiro segundo $\left(V_{E F}\right)$ foi expresso em percentual do previsto e utilizado para quantificar o grau de obstrução das vias respiratórias do paciente.

Os dados foram analisados no programa SPSS para Windows, versão 13.0. Inicialmente, foi determinada a concordância entre médico e paciente quanto ao controle da asma utilizando o índice kappa. Posteriormente, foi determinada a freqüência de pacientes com percepção inadequada do controle da asma. Em uma análise comparativa, os pacientes foram divididos em dois grupos, de acordo com a percepção do controle da asma. Desta forma, havia um grupo que concordava com o médico quanto à percepção do controle da asma (pacientes com percepção adequada) e um grupo que não concordava com o médico (pacientes com percepção inadequada). Para comparar as variáveis categóricas entre os dois grupos foi usado o teste do qui-quadrado, e para comparar as variáveis contínuas foi usado o teste de Mann-Whitney. Foi realizada análise de regressão logística, incluindo como variáveis independentes as que se relacionaram com a percepção do controle dos sintomas de asma com um $p<0,1$. Todos os testes foram bicaudais, e o $p$ considerado estatisticamente significante foi de 0,05.

\section{Resultados}

Neste estudo, foram avaliados 289 pacientes, sendo $214(74 \%)$ do sexo feminino. A mediana de idade foi de 45 anos (variação, 31-56 anos). A freqüência de pacientes alfabetizados foi de 93\%, porém apenas $29 \%$ tinham renda maior ou igual a dois salários mínimos. A avaliação da gravidade da asma demonstrou que 80 pacientes (28\%) tinham asma intermitente leve, 41 (14\%) tinham asma persistente leve, 43 (15\%) tinham asma moderada, e 125 (43\%) tinham asma grave. A mediana do tempo de sintomas de asma foi de 22 anos (variação, 8-34 anos). A mediana do $\mathrm{VEF}_{1} \%$ do previsto foi de 66\% (variação, 52-80\%). Um total de 181 pacientes (62\%) fazia uso regular de corticóide inalatório.

A Tabela 1 mostra uma baixa concordância na percepção do controle da asma entre médicos e pacientes (índice kappa $=0,5)(p<0,01)$, com 66 pacientes (23\%) apresentando percepção inadequada do controle da doença, quando comparados aos médicos. A Tabela 2 mostra as características dos pacientes agrupados de acordo com a capacidade de perceber corretamente ou não o controle da asma. Não houve diferença entre os grupos quanto à freqüência de indivíduos do sexo masculino (24 vs. 32\%) ( $p=0,22)$, freqüência de pacientes alfabetizados (94 vs. 88\%) $(p=0,46)$, freqüência do diagnóstico de rinite $(70$ vs. $68 \%)(p=0,75)$ e do diagnóstico de depressão (40 vs. 34\%) $(p=0,61)$, tempo de sintomas de asma $(p=0,82)$, escore de depressão de Beck $(p=0,6)$ ou grau de obstrução

Tabela 1 - Freqüência de pacientes com percepção inadequada do controle da asma.

\begin{tabular}{llcc}
\hline & & \multicolumn{2}{c}{$\begin{array}{c}\text { Percepção do controle } \\
\text { da asma pelo médico }\end{array}$} \\
\cline { 3 - 4 } & & $\begin{array}{c}\text { Asma não } \\
\text { controlada } \\
\mathrm{n}(\%)\end{array}$ & $\begin{array}{c}\text { Asma } \\
\text { controlada } \\
\mathrm{n}(\%)\end{array}$ \\
\hline $\begin{array}{l}\text { Percepção } \\
\text { do controle } \\
\text { da asma pelo } \\
\text { paciente }\end{array}$ & $\begin{array}{l}\text { Asma não } \\
\text { controlada } \\
\text { Asma }\end{array}$ & $153(53)$ & $39(14)$ \\
\hline
\end{tabular}


Tabela 2 - Características dos pacientes divididos de acordo com a capacidade de perceber adequadamente ou não o controle da asma.

\begin{tabular}{|c|c|c|c|c|}
\hline Variáveis & $\begin{array}{c}\text { Pacientes com percepção } \\
\text { adequada } \\
(\mathrm{n}=223)\end{array}$ & $\begin{array}{c}\text { Pacientes com percepção } \\
\text { inadequada } \\
(\mathrm{n}=66) \\
\end{array}$ & $p$ & $p^{a}$ \\
\hline Sexo masculino $(\%)^{\mathrm{b}}$ & $54(24)$ & $21(32)$ & 0,22 & \\
\hline ldade ${ }^{c}$ & $44(32-54)$ & $49(39-59)$ & $<0,01$ & 0,02 \\
\hline Tempo de sintomas de asma (anos) & $24(9-36)$ & $22(9-39)$ & 0,82 & \\
\hline Escolaridade $(\%)^{\mathrm{b}}$ & & & 0,08 & 0,46 \\
\hline Analfabeto & $13(6)$ & $8(12)$ & & \\
\hline Alfabetizado & $210(94)$ & $57(88)$ & & \\
\hline Renda familiar (\%) ${ }^{\mathrm{b}}$ & & & 0,03 & 0,02 \\
\hline$<2$ salários mínimos & $148(68)$ & $53(82)$ & & \\
\hline$\geq 2$ salários mínimos & $71(32)$ & $12(18)$ & & \\
\hline Diagnóstico de rinite $(\%)^{\mathrm{b}}$ & $152(70)$ & $44(68)$ & 0,75 & \\
\hline Escore do questionário de Beck ${ }^{c}$ & $18(11-28)$ & $15(9-24)$ & 0,60 & \\
\hline Diagnóstico de depressão $(\%)^{\mathrm{b}}$ & $51(40)$ & $12(34)$ & 0,61 & \\
\hline Gravidade da asma $(\%)^{\mathrm{b}}$ & & & $<0,01$ & $<0,01$ \\
\hline Intermitente leve & $58(26)$ & $22(33)$ & & \\
\hline Persistente leve & $20(9)$ & $21(32)$ & & \\
\hline Moderada & 37 (17) & $6(9)$ & & \\
\hline Grave & $108(48)$ & $17(26)$ & & \\
\hline $\mathrm{VEF}_{1} \%$ do previsto ${ }^{\mathrm{d}}$ & $62(47-75)$ & $68(54-79)$ & 0,66 & \\
\hline CVF \% do previsto ${ }^{\mathrm{d}}$ & $85(73-96)$ & $88(78-102)$ & 0,43 & \\
\hline
\end{tabular}

Variáveis contínuas expressas em mediana e intervalo interquartil $\left(25^{\circ}\right.$ e $75^{\circ}$ percentis); variáveis categóricas expressas por sua freqüência (número e percentual). ${ }^{a}$ Análise de regressão logística: $\mathrm{p}$ ajustado para idade, escolaridade, renda e gravidade da asma; ${ }^{\mathrm{b}}$ Qui-quadrado; cHouve 166 pacientes avaliados por meio de questionário de depressão; e ${ }^{\mathrm{d} M a n n-W h i t n e y . ~}$

de vias respiratórias $\left(\mathrm{VEF}_{1}\right)(\mathrm{p}=0,66)$. Entretanto, os pacientes com percepção inadequada tinham mais idade $(p=0,02)$, menor renda familiar $(p=0,02)$ e menor gravidade da asma $(p<0,01)$ do que os pacientes com percepção adequada do controle da asma. Em uma análise multivariada, foi possível observar que os valores de $p$ ajustados se mantiveram estatisticamente significativos. 0 estudo dos 78 pacientes com sintomas persistentes de asma que participavam do ProAR revelou que a adesão ao tratamento foi de $78 \%$ entre aqueles com percepção adequada do controle da asma e de 73\% entre aqueles com percepção inadequada $(p>0,05)$, indicando que a percepção do controle da asma não influenciou a adesão ao tratamento nesta amostra.

\section{Discussão}

Os resultados deste estudo indicam que, num ambulatório de referência, o percentual de pacientes asmáticos que não percebem adequadamente o controle da asma é significativo. A idade mais avan- çada foi um dos fatores associados à percepção inadequada do controle da asma. Alguns autores, estudando pacientes idosos, também observaram freqüência elevada de discordância entre médicos e pacientes quanto à importância dos sintomas relatados, especialmente quando os sintomas são crônicos. ${ }^{(10)}$ No presente estudo, não avaliamos crianças asmáticas. Esta também seria, provavelmente, uma população de risco para percepção inadequada do controle da asma. Uma observação interessante do nosso trabalho é que o paciente com sintomas mais graves tem melhor percepção do controle da asma. Estudos anteriores sugerem que a redução do calibre das vias aéreas não é percebida adequadamente por meio de sintomas em pacientes com asma mais grave. ${ }^{(11,12)}$ Porém, os nossos resultados fazem supor que uma vez que o sintoma é percebido, ele é corretamente entendido como um sinal de que a doença está descontrolada. Por outro lado, pacientes com asma leve subestimam ou superestimam os sintomas de asma com maior 
freqüência, talvez por terem menor experiência com o manejo da doença, tendo em vista que os sintomas são menos freqüentes nesta população. Este resultado também é compatível com a observação de que $66 \%$ dos pacientes com sintomas de asma atendidos no sistema de saúde primária (onde a freqüência de asma leve é maior) não informam a presença dos sintomas durante a consulta médica. ${ }^{(4)}$

No presente estudo não foi possivel observar associação estatisticamente significante entre escolaridade e percepção do controle da asma. Tendo em vista que o analfabetismo indica uma menor performance cognitiva, os resultados do nosso estudo contradizem os de um estudo realizado por alguns pesquisadores italianos, os quais observaram percepção inadequada de sintomas em pacientes idosos com menor performance cognitiva. ${ }^{(13)}$ É pouco provável que o tamanho da nossa amostra explique a ausência de diferença neste desfecho, uma vez que a análise post hoc indica um poder próximo de 100\% na avaliação deste aspecto. 0 mais provável é que a escolaridade não tenha influência na percepção da asma ou que os pacientes alfabetizados da população estudada também tenham baixa performance cognitiva.

Apesar de o senso comum ser o de que pacientes depressivos têm tendência a omitir sintomas ou a exagerá-los, neste estudo, não existiu associação entre depressão e percepção inadequada do controle da asma. Estudos anteriores que avaliaram a influência do humor na percepção dos sintomas de asma têm resultados controversos. Alguns autores demonstraram que uma situação de afetividade negativa poderia interferir na percepção da dispnéia, ${ }^{(14)}$ e outros observaram que pacientes com asma grave e depressão relatam menos sintomas, apesar de terem mais obstrução de vias aéreas, sugerindo que a depressão compromete a percepção do controle da asma. ${ }^{(15)}$ Entretanto, as observações de um outro grupo de pesquisadores demonstraram que o humor não influencia a percepção dos sintomas de asma. ${ }^{(16)}$

No presente estudo, os resultados também indicaram que a percepção inadequada do controle da asma nos pacientes com sintomas persistentes não influenciou a adesão ao tratamento, possivelmente porque os pacientes compreendem que o uso de medicações que previnem sintomas não deve ser descontinuado, mesmo quando os pacientes acreditam que os sintomas estão ausentes.
Os nossos resultados têm importância no manejo diário do paciente com asma pois chamam a atenção para a necessidade de uma avaliação mais cuidadosa dos pacientes idosos, com baixa renda familiar e com asma de menor gravidade. Nestes pacientes, o risco de subestimar ou superestimar a gravidade da asma é maior. Problemas com sub-medicação ou excesso de medicação podem ser evitados se o médico questionar ativamente quanto aos sintomas e ficar atendo para diferenciar sintomas que simulam asma, mas que estão relacionados a outras condições. Um exemplo é a diferenciação entre a dispnéia induzida pelo broncoespasmo e a sensação de cansaço relacionada à fadiga muscular e à falta de condicionamento físico.

A principal limitação deste estudo é a característica da população estudada. Quase a metade dos pacientes teve asma grave, o que reflete o perfil de pacientes acompanhados no sistema de saúde público terciário. No sistema de saúde primário, a maior parte dos pacientes tem asma persistente leve ou moderada. Tendo em vista que nossos resultados indicam que o paciente com doença leve tem uma percepção pior do significado dos sintomas, o médico que presta assistência na rede primária de saúde deve dar atenção ainda maior ao problema da má percepção do controle dos sintomas no paciente asmático. A realização de espirometria na avaliação inicial de todo o paciente asmático pode trazer subsídio relevante para a identificação de indivíduos que não percebem adequadamente a gravidade da sua doença.

Em conclusão, a freqüência de pacientes com asma que têm percepção inadequada do controle da doença é significativa. Atenção especial deve ser dada aos pacientes idosos, com baixa renda familiar e com doença mais leve. Estes pacientes têm maior risco de perceber o controle da doença de forma inadequada, sendo necessária uma avaliação clínica mais cuidadosa. Estudos futuros devem ser feitos para avaliar se um programa de educação em asma pode reverter esta situação.

\section{Referências}

1. Bateman ED, Boushey HA, Bousquet J, Busse WW, Clark TJ, Pauwels RA, et al. Can guideline-defined asthma control be achieved? The Gaining Optimal Asthma Control study. Am J Respir Crit Care Med. 2004;170(8):836-44.

2. Rabe KF, Vermeire PA, Soriano JB, Maier WC. Clinical management of asthma in 1999: the Asthma Insights 
and Reality in Europe (AIRE) study. Eur Respir J. 2000;16(5):802-7.

3. Gaga M, Papageorgiou N, Zervas E, Gioulekas D, Konstantopoulos S. Control of asthma under specialist care: is it achieved? Chest. 2005;128(1):78-84.

4. van Schayck CP, van Der Heijden FM, van Den Boom G, Tirimanna PR, van Herwaarden CL. Underdiagnosis of asthma: is the doctor or the patient to blame? The DIMCA project. Thorax. 2000;55(7):562-5.

5. Ponte E, Franco RA, Souza-Machado A, Souza-Machado C, Cruz AA. Impacto de um programa para o controle da asma grave na utilização de recursos do Sistema Único de Saúde. J Bras Pneumol. 2007;33(1):15-9.

6. Sociedade Brasileira de Pneumologia e Tisiologia. 111 Consenso Brasileiro no Manejo da Asma 2002. J Pneumol. 2002;28(Supl 1):S1-S151.

7. Gorenstein C, Andrade L. Validation of a Portuguese version of the Beck Depression Inventory and the State-Trait Anxiety Inventory in Brazilian subjects. Braz J Med Biol Res. 1996;29(4):453-7.

8. Gorestein C, Andrade L. Inventário de depressão de Beck: propriedades psicométricas da versão em português. Rev Psiq Clin. 1998;25(5):245-50.

9. Pereira CAC, Viegas CAA, Alves RR. Capacidade de difusão do monóxido de carbono. J Pneumol. 2002;28(Supl 3):S122-S38.
10. Scheuer E, Steurer J, Buddeberg C. Predictors of differences in symptom perception of older patients and their doctors. Fam Pract. 2002;19(4):357-61.

11. Bijl-Hofland ID, Cloosterman SG, Folgering HT, Akkermans $\mathrm{RP}$, van Schayck CP. Relation of the perception of airway obstruction to the severity of asthma. Thorax. 1999;54(1):15-9.

12. Chen E, Hermann C, Rodgers D, Oliver-Welker T, Strunk RC. Symptom perception in childhood asthma: the role of anxiety and asthma severity. Health Psychol. 2006;25(3):389-95.

13. Frisoni GB, Fedi V, Geroldi C, Trabucchi M. Cognition and the perception of physical symptoms in the communitydwelling elderly. Behav Med. 1999;25(1):5-12.

14. Bogaerts K, Notebaert K, Van Diest I, Devriese S, De Peuter $\mathrm{S}$, Van den Bergh 0. Accuracy of respiratory symptom perception in different affective contexts. J Psychosom Res. 2005;58(6):537-43.

15. Souza-Machado A, Tonheiro-Machado D, Portela PG, Fontanelle-Neto C, Cruz AA. Freqüência de depressão em pacientes ambulatoriais com asma moderada a grave. Rev Bras Alerg Imunopatol. 2001;24(4):90-7.

16. Apter AJ, Affleck G, Reisine ST, Tennen HA, Barrows E, Wells M, et al. Perception of airway obstruction in asthma: sequential daily analyses of symptoms, peak expiratory flow rate, and mood. J Allergy Clin Immunol. 1997;99(5):605-12. 